nicht nachvollziehbare Regelung. Es muss den Kostenträgern klar sein, dass solche vernünftigen Methoden nur dann eingesetzt werden, wenn sie vom Krankenhaus finanziert werden können.

CardioVasc: Der Nutzen der FFR ist in Studien belegt worden, zuletzt in FAME-2. Ihr ging die leidenschaftlich diskutierte $\mathrm{COU}$ RAGE-Studie mit der Botschaft voraus, die optimale medikamentöse Therapie sei mindestens so gut wie die PCI.

Werner: Es war ein extrem selektiertes Patientenkollektiv. Nur 6 Prozent der ursprünglich gescreenten Patienten wurden randomisiert. Und was bei der Interpretation der Ergebnisse außer Acht gelassen wurde: Bei jedem Patienten war der angiografische Befund bekannt. Das führte dazu, dass gefährdete Patienten erkannt und dann später nicht randomisiert wurden, denken Sie nur an die Hauptstammstenosen.

Die paradoxe Reaktion in den USA war die Reduktion der diagnostischen Angiografie, worum es in COURAGE gar nicht ging. Das hat nun letztlich zu der großen ISCHEMIAStudie geführt, die derzeit durchgeführt wird und die das diagnostische und therapeutische Procedere bei der stabilen KHK nochmals überprüfen wird.
CardioVasc: Ein Fortschritt war die FAME2-Studie. Fragliche Stenosen wurden einer FFR unterzogen, erst dann wurde entschieden, ob sie in die Studie aufgenommen werden. Positiver FFR und primärer Endpunkt korrelierten. Der bestand aber nicht nur aus der Gesamtmortalität.

Werner: Der Endpunkt umfasste Tod, Herzinfarkt oder Notfall-Revaskularisation. Die Gesamtmortalität ist selten der alleinige Endpunkt bei der stabilen Angina, die als benigne Verlaufsform der KHK anzusehen ist. Es geht dabei nicht um die Reduktion der Sterblichkeit, sondern um die Verbesserung der Lebensqualität, also auch um die Reduktion der einzunehmenden Medikamente und bessere Belastbarkeit im Alter.

CardioVasc: Der für das Ergebnis ausschlaggebende Punkt in FAME-2 war die Quote der Notfall-Revaskularisationen.

Werner: Den Hauptunterschied zwischen beiden Gruppen, also zwischen der PCl oder keiner PCI nach FFR-Messung, machte die notfallmäßige Revaskularisation. 50\% waren veranlasst durch einen akuten Herzinfarkt, 20\% durch einen STEMI, 30\% durch einen NSTEMI. Das sind nicht weg zu diskutierende "harte" Ereignisse und nur weitere 50\% machten die instabilen Anginen aus. Deshalb wur- de die Studie auch vorzeitig abgebrochen. Die hohe Komplikationsrate in der nur medikamentös behandelten Gruppe war nicht mehr verantwortbar.

ICardioVasc: Die FFR wird weiterentwickelt, geht es auch ohne Adenosin?

Werner: Sie sprechen die instent wave-free Flussreserve (iFR) an. Das ist eine Methode wie die FFR, aber ohne Verwendung von Adenosin. Die iFR soll mit der Computeranalyse einer bestimmten Phase des Druckkurvenverlaufs ermöglichen, eine gleiche Diskriminierung zu erzielen wie unter Adenosinbelastung. Das Verfahren ist derzeit in der Evaluierung. Wenn es funktioniert, hätte es den großen Vorteil, dass die methodischen Probleme, die mit der Adenosingabe verbunden sind (ausreichende Dosis, ausreichende Ischämie) überwunden würden und Kosten gespart werden könnten. Denn die Adenosininfusion ist durchaus auch ein Kostenfaktor. Bislang sehen die Vergleiche zwischen den Methoden vielversprechend aus. Weshalb ich noch etwas skeptisch bin: Die Grenzwerte zwischen normal und pathologisch werden verändert von 0,8 auf 0,9. Dadurch wird die Grenze sehr viel schmaler. Und je schmaler diese ist, umso anfälliger ist sie für Nebeneinflüsse.

Interview: Jochen Aumiller In

\title{
EURO-CTO-Studie
}

\section{Chronische Koronarverschlüsse eröffnen?}

Unklar ist oft, wie mittelschwere Koronarläsionen behandelt werden sollen. Aber ausgesprochen strittig ist das Prozedere bei Komplettverschlüssen: Eröffnen oder nicht? Die Argumente dagegen:

- Hinter dem Verschluss ist nach einem Infarkt alles abgestorben oder

- die Kollateralen funktionieren noch so gut, dass man keine Verbesserung erreicht, wenn man den Verschluss eröffnet.

Zunächst ist es wichtig, dass die Vitalität des versorgten poststenotischen Areals nachgewiesen wird. Die meisten chronischen Verschlüsse (CTOs) haben keine Infarktvorgeschichte. Bei nachgewiesener Vitalität wissen wir durch die Druckmessung hinter dem Verschluss, dass Kollateralen fast nie ausreichen, um ein verschlossenes Gefäß zu kompensieren. Dieser Standpunkt ist aber nicht allgemein bekannt und nicht bewusst. In Leitlinien werden CTOs immer als separate Gruppe beurteilt, in den amerikanischen Appropriateness-Guidelines sogar ganz "stiefmütterlich“. Danach dürfte man einen CTO nur behandeln, wenn ein Patient trotz medikamentöser Therapie heftige Schmerzen hat.

Damit diese Problematik ein für allemal evidenzbasiert beantwortet wird ist nun die EURO-CTO-Studie (Evaluation of Utilisation of Revascularisation for chronic total Occlusions) aufgelegt worden. Die Frage: Soll ein СTO belassen oder behandelt wer- den? Studienleiter ist Prof. Gerald Werner, Medizinische Klinik I (Kardiologie) des Klinikums Darmstadt. 1200 Patienten sollen rekrutiert werden, davon werden 400 in die Gruppe mit optimaler medikamentöser Therapie randomisiert, 800 zur interventionellen Therapie. Diese europäische Studie hat im März 2012 in 35 Zentren begonnen. Die Rekrutierungsphase soll zwei Jahre betragen. Nach einer Follow-up-Periode von einem Jahr wird Bilanz gezogen, ob es Unterschiede in der Lebensqualität gibt, der primäre Studienendpunkt. Über drei und fünf Jahre wird dann kontrolliert, wie die Sicherheitsparameter in beiden Armen ausfallen. Es könnte ja sein, dass die PCI für diese Patienten riskant ist.

(J.A.)I 